\title{
Routine clinical administration of 4-week alverine citrate and simeticone combination relief global IBS symptoms
}

\author{
Xiangping Wang ${ }^{1}$, Yikuan Feng ${ }^{2}$, Jiansheng $\mathrm{Li}^{3}$, Zibin Tian ${ }^{4}$, Dong $\mathrm{Wei}^{5}$, Lingzhi $\mathrm{Qi}^{6}$, \\ Lifeng Fang ${ }^{7}$, Kaichun $\mathrm{Wu}^{1}$ \\ ${ }^{1}$ State Key Laboratory of Cancer Biology and Xijing Hospital of Digestive Diseases, Fourth Military Medical University, Xi'an, \\ China \\ ${ }^{2}$ Gastroenterology Institute, Weifang People’s Hospital, Weifang, China \\ ${ }^{3}$ Gastroenterology Institute, 1st Affiliated Hospital of Zhengzhou University, Zhengzhou, China \\ ${ }^{4}$ Gastroenterology Institute, The Affiliated Hospital of Medical College, Qingdao University, Qingdao, China \\ ${ }^{5}$ Gastroenterology Institute, No. 150 Central Hospital of PLA, Luoyang, China \\ ${ }^{6}$ Gastroenterology Institute, The People’s Hospital of Jilin Province, Changchun, China \\ ${ }^{7}$ Gastroenterology Institute, Zhengzhou First People’s Hospital, Zhengzhou, China \\ Email: Dr_XPwang@163.com
}

Received 25 November 2013; revised 26 December 2013; accepted 9 January 2014

Copyright (C) 2014 Xiangping Wang et al. This is an open access article distributed under the Creative Commons Attribution License, which permits unrestricted use, distribution, and reproduction in any medium, provided the original work is properly cited. In accordance of the Creative Commons Attribution License all Copyrights (C) 2014 are reserved for SCIRP and the owner of the intellectual property Xiangping Wang et al. All Copyright (C) 2014 are guarded by law and by SCIRP as a guardian.

\section{ABSTRACT}

Introduction: The primary treatment aim for irritable bowel syndrome (IBS) is to relieve overall symptoms which can significantly impair the patient's quality of life (QOL); however, it generally requires a high pill burden that may be improved by administration of combinatorial formulations. Thus, the effectiveness of alverine citrate and simeticone combination (ACS) for global symptom relief for IBS was investigated in this non-interventional study. Patients and Methods: ROME III IBS patients $(n=640$; $52.3 \%$ male: mean age: $43.6 \pm 12.5$ years) with abdominal pain and discomfort $\geq 60$ of 0 - 100 visual analogue scale (VAS) were included in a prospective, multicenter, non-interventional study at 26 Chinese sites from December 2010 to January 2012. Patients received alverine citrate $(60 \mathrm{mg})$ with simeticone $(300$ mg) (ACS) $3 \times$ daily for 4 weeks. Pain/discomfort and bloating/distension were assessed by VAS. Global symptoms and QOL were assessed by 7-point and 5-point Likert scales, respectively. Post-treatment bowel function was assessed by Bristol Stool Form Scale (BSFS) and treatment-related adverse events (AEs) were recorded. Results: Of 640 patients, 540 (84.4\%) completed the study, and 100 (15.6\%) withdrew. In total, $87.5 \%$ reported bloating at baseline. After 4-week ACS treatment, 89.1\% reported global symptom improvement. Furthermore, 4-week ACS treatment reduced pain and bloated VAS scores sig- nificantly from $78.4 \pm 9.9$ to $32.1 \pm 21.0$ and from 63.2 \pm 27.2 to $22.6 \pm 20.9$, respectively (both $p<0.001$ ), decreased diarrhea or constipation occurrence from $67.2 \%$ to $10.2 \%(p<0.001)$, and reduced IBS impact on QOL with only 2 treatment-related AEs. Conclusion: Routine clinical administration of ACS for IBS over a 4-week period provides effective relief of IBS symptoms and improves QOL in IBS patients.

\section{KEYWORDS}

Alverine Citrate; Abdominal Pain; Bloating; Irritable Bowel Syndrome; Simeticone

\section{INTRODUCTION}

Irritable bowel syndrome (IBS) is a symptomatic motility and sensory disorder of the lower gastrointestinal (GI) tract leading to a wide array of symptoms, including abdominal pain and discomfort, bloating, distension, and altered bowel function [1]. Many patients experience reduced quality of life (QOL) as a result of sporadic acute periods of severe symptoms, colloquially referred to as IBS "flare ups" [2]. In western countries, the prevalence of IBS ranges $7 \%$ to $10 \%$ in general population, and recent epidemiological data in China have suggested that IBS occurs in $5.7 \%$ and $7.9 \%$ of individuals in normal communities and colleges/universities, respectively $[3,4]$. In affected patients and populations, IBS imposes a substantial and intensely negative socioeconomic burden, 
with $68 \%$ of IBS patients reporting to have missed work or social functions more than 10 times in a 3-month period [5]. As a result of low cost-efficacy and poor toleration of treatments, many patients report loss of productivity, higher healthcare utilization, and high out-ofpocket medical costs as well as increased anxiety and abdominal surgery rates [5]. Thus, there is an urgent need for more cost-effective, tolerable and efficacious treatments for IBS.

The pathophysiology of IBS has been linked with a variety of mechanisms, including abnormal gastrointestinal (GI) motor function, enhanced visceral perception, brain-gut axis dysfunction, intestinal dysbiosis, micro-inflammatory changes, and psychological disturbances. As a result, no unidimensional treatment exists [6], and variant outcomes have been reported with a variety of conventional medicinal treatments that target specific symptoms (e.g. laxatives, antispasmodics, antidiarrheal, serotoninergic agents) as well as psychotherapy treatment strategies [5]. Thus, the development and assessments of combination therapies may be central to providing global symptom relief for IBS patients.

The most common medicinal regimens for IBS include a combination of antispasmodics for abdominal pain, dimeticone or simeticone for bloating, and anti-diarrheal or laxative drugs to improve bowel habit disturbances $[7,8]$. However, not only do these treatments tend to leave some significant symptoms untreated, but also the pill burden on patients is high, causing issues with patient compliance that affects treatment outcomes [9]. Thus, novel combination formulations may notably reduce the pill burden, achieving better outcomes and patient compliance improvement.

Combined alverine citrate and simeticone (ACS) (Meteospasmyl ${ }^{\circledR}$ ) is an orally-administrated capsule that combines $60 \mathrm{mg}$ of alverine citrate, an active substance derived from Papaverine, and 300 mg simeticone, dimeticone enriched with silicon dioxide. Alverine citrate has been reported to exhibit a dual intestinal pharmacological activity with few systemic side effects [10]. It exerts a spasmolytic effect on smooth muscle cells that acts through basal and stimulated motility via a calcium-dependent and -independent inhibition of neuronal excitability [11] and through smooth muscle cell L-type $\mathrm{Ca}^{2+}$ channels [12]. Additionally, alverine citrate has an antinociceptive effect associated with $5-\mathrm{HT}_{1 \mathrm{~A}}$ receptor antagonism, allowing it to modulate nociceptive response and visceral hypersensitivity [10]. Simeticone is an inert hydrophobic drug with an anti- foaming effect that acts to reduce flatulence [13]. Latest publication also suggested a synergistic action of these 2 compounds on visceral pain decreasing stress-induced colonic permeability [14].

The clinical efficacy of ACS for treating abdominal pain and discomfort has been reported in some clinical studies, and ACS has been compared to treatment with other conventional antispasmodic agents, such as Mebeverine, Trimebutine and Pinaverium, in terms of ability to effectively relieve pain and discomfort [15-17]. The majority of studies of ACS for use in treatment, however, predominantly reported its ability to relieve pain, with minimal or no consideration for the ability of ACS to reduce other symptoms of IBS, such as bloating, flatulence, and overall impact on QOL. The current non-interventional study was conducted to comprehensively investigate the effects of ACS treatment on a broad array of IBS symptoms and overall effect on QOL, thereby providing useful evidence for the applicability of this combinatorial drug for routine clinical treatment of IBS patients.

\section{PATIENTS AND METHODS}

\subsection{Study Design}

A multicenter, non-interventional study was conducted at 26 gastroenterological sites in China from December 2010 to January 2012. The final protocol was approved independently by the Ethics Committee of each participating hospital, and the study was conducted in accordance with the International Conference on Harmonisation (ICH) Guidelines for Good Clinical Practice, the Declaration of Helsinki, and applicable local regulations. All patients provided written informed consent for participation.

\subsection{Patient Selection}

Patients were included that were of adult age ( $\geq 18$ years), had a current diagnosis of IBS according to the ROME III criteria [18], reported intensity of abdominal pain and discomfort $\geq 60$ for the preceding 7-day period according to Visual Analogical Scale (VAS) assessments, and were currently receiving treatment for IBS with ACS. Patients were excluded that exhibited non-IBS diagnoses; presented alarm signs or symptoms of other conditions, such as anemia, rectal bleeding, unexplained weight loss, or general health status deterioration; exhibited severe constipation, defined as $\leq 1$ stool/week; had a history of gastro-intestinal cancer or gastro-intestinal surgery or acute/uncontrolled systemic pathology; or presented with a known contraindication to ACS treatment, such as intolerance of individual ACS components or lactose intolerance.

\subsection{Drug Treatment}

During the observational period, all patients were administrated ACS (alverine citrate $60 \mathrm{mg}$ and simeticone 300 mg; Meteospasmyl ${ }^{\circledR}$, Mayoly-Spindler, France) 3 capsules daily. All capsules were administered prior to 
meals at approximately the same times every day. Concomitant therapies were permitted, but were recorded.

\subsection{Data Collection}

Examinations were scheduled according to the start date of ACS treatment at baseline (week 0), week 2, and week 4. During each examination, patients self-reported abdominal pain/discomfort and abdominal bloating/distention experienced in the preceding 7-day period on a 0 100 VAS scale. Stool assessment (weekly average frequency and most frequent type) were performed by using the Bristol Stool Form Scale (BSFS). Global assessment of symptoms was conducted using a 7-point Likert scale both by physicians and patients. Patients also quantified the impact of IBS on their QOL by responding to the following statement: "My bowel problems limit my life and daily activities" using a 5-point Likert scale, with scores ranging from 1 to 5, as follows: "extremely", “quite a bit”, "moderately”, “a little” or "not at all”. Furthermore, patients made daily self-report of concomitant therapy use; and compliance with the study drug protocol.

\subsection{Efficacy and Safety Endpoints}

The primary efficacy endpoint was the difference in pain VAS scores from baseline to week 4. In addition, a minimum of $30 \%$ or $50 \%$ improvement in pain at week 4 compared to baseline was used to subdivide patients as responders or significant responders, respectively.

Secondary efficacy endpoints included differences in bloating VAS scores from baseline to week 2 and week 4, differences in pain VAS scores from baseline to week 2, global assessment of symptom relief (overall assessment was defined as decreased by at $\geq 1$ grade compared to baseline values), IBS impact on QOL, and alterations in remaining IBS symptoms compared to the baseline.

All spontaneous adverse events (AEs) considered as possibly related to the study treatment by the investigator, were collected during the study.

\subsection{Statistical Analysis}

All analyses were performed using SPSS version 17.0 (SPSS, Inc., Chicago, IL, USA). Analyses were performed on the full analysis set (FAS) and per protocol set (PPS). FAS included all enrolled patients administered $\geq 1$ dose of ACS with $\geq 1$ measurable criterion following treatment. FAS population corresponded to recommended intention-to-treat (ITT) population. PPS included all subjects without major protocol deviations, as follows: treatment compliance (80\% - 120\%) and visit window compliance ( \pm 2 days for each visit). All data were presented as means \pm standard deviation (SD). VAS percent change was analyzed using one sample t-test. Wilcoxon signed-rank test was used to evaluate Likert scale change. Exact confidence intervals (CIs) were constructed for binomial variables. For stool assessment, Pearson's chisquare tests were used to evaluate stool types and symptom distribution by examination time and group. Wilcoxon signed-rank test was used to analyze stool frequency variation. All tests were two-sided, and p-values less than or equal to 0.05 were considered statistically significant $(\mathrm{p} \leq 0.05)$.

\section{RESULTS}

\subsection{Patient Characteristics}

Of the 640 patients enrolled, 540 (84.4\%) completed the study, whereas 100 (15.6\%) withdrew prematurely. Most common reasons for study discontinuation included: withdrawal of informed consent (46 patients), lack of efficacy (26 patients), and loss to follow-up (11 patients). Baseline patient characteristics of ITT populations are displayed in Table 1. Mean age was $43.6 \pm 12.5$ years old and male to female ratio was 1.09:1.441 (68.9\%) patients were in the PPS. The most frequently occurring protocol violations involved treatment compliance (131 cases). No significant differences were observed between IIT and PPS populations in terms of baseline characteristics $(\mathrm{p}>0.05)$.

Table 1. Baseline demographic and clinical characteristics.

\begin{tabular}{|c|c|}
\hline & Total $(\mathrm{N}=640)$ \\
\hline Age (years) ${ }^{*}$ & $43.6 \pm 12.5$ \\
\hline Female $^{\#}$ & $305(47.7 \%)$ \\
\hline IBS duration (years, median) & 2 \\
\hline Number of days with symptoms/month ${ }^{*}$ & $13.8 \pm 7.0$ \\
\hline \multicolumn{2}{|l|}{ Associated complaints ${ }^{\#}$} \\
\hline Bloating & $560(87.5 \%)$ \\
\hline Bowel habit disorders & 294 (45.9) \\
\hline Pain/discomfort Improved after defecation & $620(96.9)$ \\
\hline Abdominal pain/discomfort (VAS) ${ }^{*}$ & $78.4 \pm 9.9$ \\
\hline Abdominal bloating/distension (VAS) ${ }^{*}$ & $63.2 \pm 27.2$ \\
\hline Stool frequency ${ }^{*}$ & $2.6 \pm 1.5$ \\
\hline \multicolumn{2}{|l|}{ Stool type (BSFS) } \\
\hline $1-2$ & $12.0 \%$ \\
\hline $6-7$ & $55.2 \%$ \\
\hline Treated for IBS within preceding 3 months ${ }^{\#}$ & $45(7.0)$ \\
\hline
\end{tabular}

\footnotetext{
* Data are presented as means $\pm \mathrm{SD}$; ${ }^{*}$ Data are presented as $\mathrm{n}(\%)$.
} 


\subsection{Treatment Exposure}

Of the total study group ( $n=640), 429(67.0 \%)$ patients were treated with ACS monotherapy and 211 (33.0\%) patients were treated with ACS plus at least one other concomitant medication, most commonly probiotics (19.2\%), traditional Chinese medicines (TCM) (6.1\%), and antispasmodics (5.8\%). During the study, the average ACS treatment compliance, which was calculated based on the actual drug taken recorded on the patient dairy, was $93.1 \% \pm 8.7 \%$, ranging from $42.4 \%$ to $156.9 \%$.

\subsection{Pain and Discomfort Relief}

Pain VAS scores decreased from $78.4 \pm 9.9$ to $52.8 \pm$ 18.7 after 2 weeks of ACS treatment, further declining to $32.1 \pm 21.0$ by week 4 . Statistically significant changes in median pain VAS scores of -22 at week 2 and -47 at week 4, respectively, were reported (both $\mathrm{p}<0.001$ ) (Figure 1(a)). By the end of week 4, responder rates and significant responder rates were $84.3 \%$ and $62.8 \%$, respectively.

\subsection{Bloating and Distension Relief}

Bloating VAS scores decreased from $63.2 \pm 27.2$ to 40.0 \pm 24.4 after 2 weeks of ACS treatment, further declining to $22.6 \pm 20.9$ by week 4 . Statistically significant
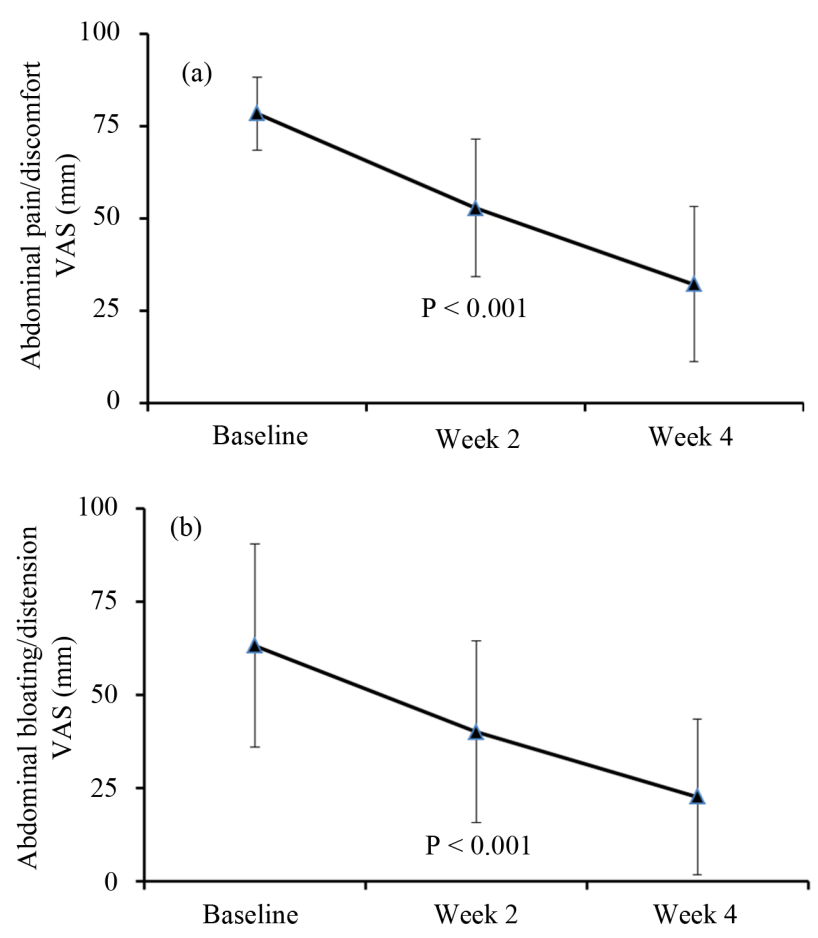

Figure 1. Progressive change in abdominal pain/discomfort and bloating/distention assessments by VAS. A: abdominal pain and discomfort; B: abdominal bloating and distention. changes in median bloating VAS scores were -19 at week 2 and -40 at week 4 , respectively (both $\mathrm{p}<0.001$ ) (Figure 1(b)).

\subsection{Stool Assessments}

Stool frequency significantly decreased from $2.6 \pm 1.5$ times/day to $1.4 \pm 0.7$ times/day from baseline to the end of week 4 ( $p<0.001)$. Number and rate of patients reporting diarrhea as the most frequent stool type during the preceding 7-day period (scores of 6 or 7) decreased throughout the study period, as follows: 353 (55.2\%) at baseline, 134 (23.4\%) at week 2, and 48 (8.9\%) at week 4. Number and rate of patients reporting constipation (scores of 1 or 2) decreased throughout the study period, as follows: $97(12.0 \%)$ at baseline, $16(2.8 \%)$ at week 2, and $7(1.3 \%)$ at week 4 (Table 2). Additionally, nonspecified bowel complaints, including straining, urgency, and feeling of incomplete defecation, were also significantly improved (Figure 2).

Table 2. Change in BSFS stool type in the ITT population.

\begin{tabular}{|c|c|c|c|}
\hline $\begin{array}{l}\text { BSFS } \\
\text { Type }^{*}\end{array}$ & $\begin{array}{l}\text { Week } 0 \text { (baseline) } \\
\qquad(\mathrm{N}=640)\end{array}$ & $\begin{array}{c}\text { Week } 2 \\
(\mathrm{~N}=572)\end{array}$ & $\begin{array}{l}\text { Week } 4 \\
(\mathrm{~N}=541)\end{array}$ \\
\hline 1 & 30 (4.7\%) & 0 & 0 \\
\hline 2 & 47 (7.3\%) & $16(2.8 \%)$ & $7(1.3 \%)$ \\
\hline 3 & $53(8.3 \%)$ & 69 (12.1\%) & $26(4.8 \%)$ \\
\hline 4 & 50 (7.8\%) & 115 (20.1\%) & $244(45.1 \%)$ \\
\hline 5 & 107 (16.7\%) & 238 (41.6\%) & 216 (39.9\%) \\
\hline 6 & $284(44.4 \%)$ & 127 (22.2\%) & 48 (8.9\%) \\
\hline 7 & 69 (10.8\%) & $7(1.2 \%)$ & 0 \\
\hline
\end{tabular}

* The most frequent stool type during the last 7 days.

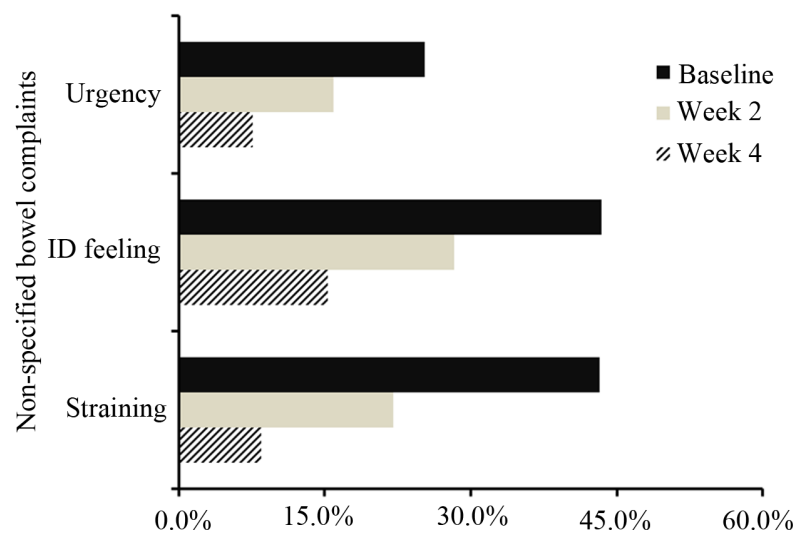

Figure 2. Change in non-specified bowel complaints from baseline (week 0) to week 4. ID means incomplete defecation. 


\subsection{Global Assessment of IBS Symptoms}

After 2 weeks of ACS treatment, self-reported and clinically-measured responder and significant responder rates were $67 \%$ and $69.6 \%$, respectively. These values increased to $89.1 \%$ and $90.0 \%$, respectively, after 4 weeks of ACS treatment. No significant differences were reported in the positive global assessments reported by investigators during clinical examinations and patient self-reports $(p>0.05)$.

\subsection{IBS Impact on QOL}

The impact of IBS on QOL scores improved progressively throughout the 4-week treatment period, indicating reduced impact of IBS on functions involved in QOL (Table 3). Responder rates increased from $65.2 \%$ at week 2 to $84.7 \%$ at week 4 . No significant differences were observed between IIT and PPS population regarding above efficacy endpoints ( $p>0.05)$.

\subsection{Safety of ACS Treatment}

During the whole study period, the only treatment-related AEs reported were tremors in limb muscle $(n=1)$ and chest distress $(n=1)$ from the same patient on the 7th day after treatment. Symptoms related to these AEs disappeared after drug withdrawal without requiring other intervention. No deaths or other treatment-related serious adverse events (SAEs) were reported.

\section{DISCUSSION}

ACS was demonstrated to effectively relieve global IBS symptoms, including abdominal pain and discomfort, bloating and distension, and other bowel habit disturbances in Chinese IBS patients fulfilling the ROME III criteria. Furthermore, a 4-week course of ACS was shown to be highly effective and generally safe, with no

Table 3. IBS impact on QOL in the ITT population from baseline to week 4.

\begin{tabular}{cccc}
\hline Grade & $\begin{array}{c}\text { Baseline } \\
(\mathrm{N}=640)\end{array}$ & $\begin{array}{c}\text { Week 2 } \\
(\mathrm{N}=572)\end{array}$ & $\begin{array}{c}\text { Week 4 } \\
(\mathrm{N}=541)\end{array}$ \\
\hline 1-Extremely & $72(11.3)$ & $4(0.7)$ & $1(0.2)$ \\
2—Quite a bit & $337(52.7)$ & $86(15.0)$ & $19(3.5)$ \\
3-Moderately & $136(21.3)$ & $274(47.9)$ & $120(22.2)$ \\
4-A little & $91(14.2)$ & $186(32.5)$ & $248(45.8)$ \\
5-Not at all & $4(0.6)$ & $22(3.8)$ & $153(28.3)$ \\
Mean \pm SD & $2.4 \pm 0.9$ & $3.2 \pm 0.8^{*}$ & $4.0 \pm 0.8^{*}$ \\
\hline
\end{tabular}

${ }^{*} \mathrm{P}<0.0001$ vs. baseline (week 0). occurrence of death or SAEs and 2 relatively mild AEs reported. Thus ACS appears to be appropriate for routine clinical treatment of IBS patients.

Clinical studies [15-17] that have assessed ACS, a relatively new combination drug for IBS management, have reported endpoints that indicate reductions in abdominal pain/discomfort, the key symptom of IBS, and are widely used as the primary endpoint of IBS treatment due to the lack of objective hallmark of the disease [19]. While these findings are consistent with the present results, the current study provides a much more comprehensive assessment of the effects of ACS treatment in IBS patients over the course of 2 to 4 weeks. Moreover, this comprehensive study further suggests that ACS may limit main symptoms of IBS, potentially reducing the need for additional concomitant medications.

IBS mainly generates subjective symptoms that lend pretty well to current theories of the placebo effect, namely that psychological intervention may play an important role in the treatment and that placebos may alter symptoms by affecting psychological perception of the disease [20]. This is consistent with the results of psychotherapy in IBS patients [5], suggesting that IBS has both physiological and psychological components. Wittmann et al. reported that response to ACS treatment exceeded that of patients treated by placebo by 46.8 to $34.3 \%$, respectively [22]. In fact, studies involving patients with IBS have among the highest placebo response rates, ranging from $16 \%$ to $71.4 \%$ in various studies [21-23]. A recent systematic review indicated that frequent dosing correlates with higher levels of placebo response in IBS patients; however, studies based on ROME criteria for study eligibility have much lower placebo response rates [22]. Due to the nature of this study, though placebo effects can be assumed to be minimized by employing the ROME III criteria, the placebo effect may still exist. While this effect is difficult to quantify, it may partially explain the $10 \%$ higher effectiveness of ACS on pain relief in the current study compared to the report by Wittmann et al. [22]. On the other hand, our study data may reflect the actual responder rate in real clinical practice, and could be referenced by physicians in their routine clinical practice, that is indeed important but has never been observed and reported.

Bloating has been reported to occur up to $96 \%$ of patients with functional gastrointestinal disorders [24], consistent with the current findings of $87.5 \%$ in IBS patients complaining from bloating at baseline. In a comparative study [25], ACS was revealed to exhibit similar treatment efficacy as Pinaverium bromide, a widely employed conventional antispasmodics agent, for pain relief; however, ACS also relieved abdominal bloating/distension to a much greater degree. In the study, a significant bloating/distension relief was observed during the 4- 
week treatment by VAS.

A large percentage of IBS patients reported abnormal bowel movement, especially diarrhea at baseline. These findings are generally consistent with a recent multicenter epidemiological study of Chinese IBS patients that indicated that, among 735 IBS patients meeting the ROME III criteria, $66.3 \%$ had diarrhea, $14.7 \%$ had constipation, $4.2 \%$ had both diarrhea and constipation, and $14.8 \%$ had other forms of impaired bowel movements [26]. Because chronic diarrhea is an indication that often necessitates referral from primary care to a gastroenterologist, selection of a larger number of referred patients may explain the discrepancies between the current patient population and those previously reported. Besides, previous study has indicated that small colonic diameter and accelerated small bowel transit may play a role in determining the severity of bowel dysfunction in IBS, which may effectively be treated with antispasmodics [27]. In the study, the improvement of abnormal bowel movement and non-specified bowel complaints, including straining, urgency, and feeling of incomplete defecation, were observed after 4 week ACS treatment.

Overall, minimizing the use of concomitant therapies in favor of combined medications, such as ACS, may be useful in improving global symptoms and patient compliance, with approximately $85 \%$ of patients in the current study reporting a positive impact on QOL. By improving the main IBS related symptoms, ACS may limit the need for additional drugs for symptomatic relief and be effectively incorporated into graded treatment strategies for optimal IBS symptom relief, as previously suggested [7]. In fact, the current study reported that only $33 \%$ of patients required additional medications other than ACS for satisfactory symptom relief, though there was no clear trend in the type of concomitant medications required. While probiotics were most common in the current study and previously demonstrates to be effective in IBS patients [28], further research is required to determine the most effective concomitant therapies and to determine the true benefit of probiotics, many of which are not regulated by the China Food and Drug Administration (CFDA). Furthermore, concurrent administration of traditional Chinese medicine (TCM), a routine practice in many Chinese clinical settings when western medicine fails to provide adequate symptoms relief [29], merits further study in China. Interestingly, the current study also exhibited much lower administration of anxiolytics and antidepressants (5.3\%) than other contemporary studies, including the $19 \%$ reported by Wittmann et al. [17]. These findings may potentially indicate variations in treatment practices between locations, though this and the true effects of these treatments in combination with ACS will require further study.

The current study must, however, be considered in terms of the relatively short term of the study and limited geographic population, which may not be widely applicable to patients in other regions. Furthermore, the PPS population accounted for only $69 \%$ of the total enrolled ITT population, a relatively low percentage. Further study will, however, be required to determine why such a large proportion of patients violated treatment or examination protocols; in fact, the underlying reasons may be closely related to IBS treatment compliance issues. Additionally, further long-term studies will be required, because treatment for IBS is generally ongoing, lasting longer than 2 to 4 weeks in routine clinical settings. In fact, the United States FDA currently recommends a minimum treatment of 8 weeks [30]. Whether extended treatment with ACS can offer sustained benefits is yet to be determined, meriting further investigation.

\section{CONCLUSION}

The current study demonstrated that ACS is safe and effective for global symptoms relief in patients with IBS over a 2 - 4-week treatment period. Unlike its predecessors, this study provides a comprehensive account of the well-described ability of ACS to relieve abdominal pain/ discomfort, as well as its ability to limit other symptoms (Bloating/distention, abnormal bowel habit) often reported by IBS patients following the 4-week treatment course. Thus, ACS may be beneficially employed as a routine therapeutic for IBS patients in clinical practice, potentially reducing the number of concomitant therapies required to maximally alleviate symptoms.

\section{ACKNOWLEDGEMENTS}

The study was sponsored and funded by Mayoly Spindler, France. and Beaufour-Ipsen (Tianjin) Pharmaceutical Co. Ltd, China.

\section{REFERENCES}

[1] Tack, J., Fried, M., Houghton, L.A., Spicak, J. and Fisher, G. (2006) Systematic review: The efficacy of treatments for irritable bowel syndrome-A European perspective. Alimentary Pharmacology \& Therapeutics, 24, 183-205. http://dx.doi.org/10.1111/j.1365-2036.2006.02938.x

[2] Frank, L., Kleinman, L., Rentz, A., Ciesla, G., Kim, J.J. and Zacker, C. (2002) Health-related quality of life associated with irritable bowel syndrome: Comparison with other chronic diseases. Clinical Therapeutics, 24, 675689; discussion 674. http://dx.doi.org/10.1016/S0149-2918(02)85143-8

[3] Dong, Y.Y., Zuo, X.L., Li, C.Q., Yu, Y.B., Zhao, Q.J. and Li, Y.Q. (2010) Prevalence of irritable bowel syndrome in Chinese college and university students assessed using ROME III criteria. World Journal of Gastroenterology, 16, 4221-4226. http://dx.doi.org/10.3748/wjg.v16.i33.4221

[4] Xiong, L.S., Chen, M.H., Chen, H.X., Xu, A.G., Wang, 
W.A. and Hu, P.J. (2004) A population-based epidemiologic study of irritable bowel syndrome in Guangdong province. Zhonghua Yi Xue Za Zhi, 84, 278-281.

[5] Hulisz, D. (2004) The burden of illness of irritable bowel syndrome: Current challenges and hope for the future. Journal of Managed Care Pharmacy, 10, 299-309.

[6] Ford, A.C. and Talley, N.J. (2012) Irritable bowel syndrome. BMJ, 345, e5836. http://dx.doi.org/10.1136/bmj.e5836

[7] Gastrointestinal Motility Group, Chinese Society of Gastroenterology, Chinese Medical Association (2008) Consensus on management of irritable bowel syndrome (2007 Edition, Changsha). Chinese Journal of Digestion, 28, 38-40.

[8] Spiller, R., Aziz, Q., Creed, F., Emmanuel, A., Houghton, L., Hungin, P., Jones, R., Kumar, D., Rubin, G., Trudgill, N. and Whorwell, P. (2007) Guidelines on the irritable bowel syndrome: Mechanisms and practical management. Gut, 56, 1770-1798.

http://dx.doi.org/10.1136/gut.2007.119446

[9] Drossman, D.A., Morris, C.B., Schneck S., Hu, Y.J., Norton, N.J., Norton, W.F., et al. (2009) International survey of patients with IBS: symptom features and their severity, health status, treatments, and risk taking to achieve clinical benefit. Journal of Clinical Gastroenterology, 43, 541-550.

http://dx.doi.org/10.1097/MCG.0b013e318189a7f9

[10] Coelho, A.M., Jacob, L., Fioramonti, J. and Bueno, L. (2001) Rectal antinociceptive properties of alverine citrate are linked to antagonism at the $5-\mathrm{HT}_{1 \mathrm{~A}}$ receptor sub-type. Journal of Pharmacy and Pharmacology, 53, 1419-1426. http://dx.doi.org/10.1211/0022357011777783

[11] Bouvier, M., Grimaud, J.C., Abysique, A. and Chiarelli, P. (1992) Effects of alverine on the spontaneous electrical activity and nervous control of the proximal colon of the rabbit. Gastroentérologie Clinique et Biologique, 16, 334-338.

http://www.ncbi.nlm.nih.gov/nlmcatalog/?term=gastroent erol\%20clin\%20biol\%20journal

[12] Hayase, M., Hashitani, H., Suzuki, H., Kohri, K. and Brading, A.F. (2007) Evolving mechanisms of action of alverine citrate on phasic smooth muscles. British Journal of Pharmacology, 152, 1228-1238. http://dx.doi.org/10.1038/sj.bjp.0707496

[13] Brecević, L., Bosan-Kilibarda, I. and Strajnar, F. (1994) Mechanism of antifoaming action of simethicone. Journal of Applied Toxicology, 14, 207-211. http://dx.doi.org/10.1002/jat.2550140311

[14] Bueno, L., Beaufrand, C., Theodorou, V. and AndroDelestrain, M.C. (2013) Influence of simethicone and alverine on stress-induced alterations of colonic permeability and sensitivity in rats: Beneficial effect of their association. Journal of Pharmacy and Pharmacology, 65, 567673. http://dx.doi.org/10.1111/jphp.12021

[15] Barthet, M., Mambrini, P., Salducci, J., Bouvier M., Chiarelli, P., Grimaud J.C., et al. (1996) Efficacité de l'association citrate d'alvérine+siméthicone dans le traitement du syndrome de l'intestin irritable. Les Actualités en Gastroentérologie, 10, S1-S7.
http://www.ncbi.nlm.nih.gov/nlmcatalog/?term=Act+Me d+Int+Gastroentérologie

[16] Danne, O., Pospai, D. and Mignon, M. (1996) Efficacité comparée du citrate d'alvérine (nouvelle formule) et de la mébévérine chez les adultes atteints de troubles fonctionnels intestinaux. Le Concours Médical, 118, I-VIII.

[17] Wittmann, T., Paradowski, L., Ducrotté, P., Bueno, L. and Andro Delestrain, M.C. (2010) Clinical trial: The efficacy of alverine citrate simeticone combination on abdominal pain discomfort in irritable bowel syndrome a randomized, double-blind, placebo-controlled study. Alimentary Pharmacology \& Therapeutics, 31, 615-624. http://dx.doi.org/10.1111/j.1365-2036.2009.04216.x

[18] ROME Foundation (2012) ROME III diagnostic criteria for functional gastrointestinal disorders. Appendix A: ROME III diagnostic criteria for FGIDs. http://www.romecriteria.org/assets/pdf/19 RomeIII apA 885-898.pdf

[19] Hammerle, C.W. and Surawicz, C.M. (2008) Updates on treatment of irritable bowel syndrome. World Journal of Gastroenterology, 14, 2639-2649. http://dx.doi.org/10.3748/wjg.14.2639

[20] Rajagopal, S. (2006) The placebo effect. The Psychiatrist, 30, 185-188.

[21] Pitz, M., Cheang, M. and Bernstein, C.N. (2005) Defining the Predictors of the Placebo Response in Irritable Bowel Syndrome. Clinical Gastroenterology and Hepatology, 3, 237-247. http://dx.doi.org/10.1016/S1542-3565(04)00626-3

[22] Patel, S.M., Stason, W.B., Legedza, A., Ock, S.M., Kaptchuk, T.J., Conboy, L., et al. (2005) The placebo effect in irritable bowel syndrome trials: A meta-analysis. Neurogastroenterology \& Motility, 17, 332-340. http://dx.doi.org/10.1111/j.1365-2982.2005.00650.x

[23] Dorn, S.D., Kaptchuk, T.J., Park, J.B., Nguyen, L.T., Canenguez, K., Nam, B.H., et al. (2007) A meta-analysis of the placebo response in complementary and alternative medicine trials of irritable bowel syndrome. Neurogastroenterology \& Motility, 19, 630-637.

http://dx.doi.org/10.1111/j.1365-2982.2007.00937.x

[24] Houghton, L.A. and Whorwell, P.J. (2005) Towards a better understanding of abdominal bloating and distension in functional gastrointestinal disorders. Neurogastroenterology \& Motility, 17, 500-511. http://dx.doi.org/10.1111/j.1365-2982.2005.00666.x

[25] Zhong, Y.Q., Liu, J., Li, S.S. and Zen, Z.Y. (2009) A randomized controlled study of compound alverine citrate in treating patients with diarrhea-predominant irritable bowel syndrome. Chinese Journal of Gastroenterology, 14, 324-327.

[26] Yao, X., Yang, Y.S., Cui, L.H., Zhao, K.B., Zhang, Z.H., Peng, L.H., et al. (2012) Subtypes of irritable bowel syndrome on ROME III criteria: A multicenter study. Journal of Gastroenterology and Hepatology, 27, 760-765. http://dx.doi.org/10.1111/j.1440-1746.2011.06930.x

[27] Ford, A.C. (2009) Alverine citrate, simeticone, and ROME III irritable bowel syndrome. Alimentary Pharmacology \& Therapeutics, 31, 767-768; author reply 768769. http://dx.doi.org/10.1111/j.1365-2036.2009.04222.x 
[28] McFarland, L.V. and Dublin, S. (2008) Meta-analysis of probiotics for the treatment of irritable bowel syndrome. World Journal of Gastroenterology, 14, 2650-2661. http://dx.doi.org/10.3748/wjg.14.2650

[29] Shi, J., Tong, Y., Shen, J.G. and Li, H.X. (2008) Effectiveness and safety of herbal medicines in the treatment of irritable bowel syndrome: A systematic review. World
Journal of Gastroenterology, 14, 454-462. http://dx.doi.org/10.3748/wjg.14.454

[30] Mangel, A.W. and Fehnel, S.E. (2011) End points in irritable bowel syndrome. Expert Review of Gastroenterology \& Hepatology, 5, 293-295.

http://dx.doi.org/10.1586/egh.11.13 